\title{
Why does wurtzite form in nanowires of III-V zinc-blende semiconductors?
}

\author{
Frank Glas * Jean-Christophe Harmand, and Gilles Patriarche \\ CNRS - Laboratoire de Photonique et de Nanostructures, route de Nozay, 91460 Marcoussis, France
}

\begin{abstract}
We develop a nucleation-based model to explain the formation of the wurtzite (WZ) phase during the vapor-liquid-solid growth of free-standing nanowires of zinc-blende (ZB) semiconductors. Nucleation occurs preferentially at the edge of the solid/liquid interface, which entails major differences between ZB and WZ nuclei. Depending on the pertinent interface energies, WZ nucleation is favored at high liquid supersaturation. This explains our systematic observation of ZB during early growth.
\end{abstract}

PACS numbers: 68.65.La,64.60.Qb,81.05.Ea,81.15.Kk,64.70.Nd

Free-standing wires with diameters ranging from hundreds down to a few nanometers are nowadays commonly fabricated from a large range of semiconductor materials [1, 2, 3, 4, 5]. These nanowires (NWs) have remarkable physical properties and many potential applications. The present work deals with the epitaxial growth of NWs of III-V semiconductors on a hot substrate. Metal catalyst nanoparticles deposited on the substrate before growth define the wire diameter. According to the vapor-liquidsolid (VLS) growth mechanism, the atoms are fed from the vapor phase to the solid wire through this particle (or droplet), which remains liquid during growth [6].

We consider III-V compounds which, under bulk form, adopt the cubic zinc-blende (ZB) crystal structure [7] (although some non-ZB high-pressure phases [8] may be metastable at atmospheric pressure [9]), leaving aside nitrogen-based NWs. We discuss the usual case of NWs grown on a [111]B (As-terminated) face of the ZB substrate. Probably the most surprising feature of these NWs is that, in contrast to their bulk counterparts, they often adopt the hexagonal wurtzite (WZ) structure. This was observed for most ZB III-V materials and growth techniques [1, 3, 4, 10, 11]. However, although often dominantly of WZ structure, the NWs usually contain stacking faults (SFs) and sequences of ZB structure. The coexistence of two phases is clearly a problem for basic studies as well as applications, so that phase purity control is one of the main challenges of III-V NW fabrication.

The surprising prevalence of the WZ structure in III-V NWs has not been explained satisfactorily so far. Here, based on new experimental observations, we propose an explanation of the occurrence of the WZ structure and develop a model predicting quantitatively in which growth conditions it should form. We consider the specific case of gold-catalyzed GaAs NWs grown by molecular beam epitaxy (MBE) on a GaAs substrate but we expect our model and our conclusions to remain valid for any ZB III-V compound and any growth method.

Let us start with briefly reviewing previously proposed explanations. Calculations give the difference $\delta w$ in cohesive energy between ZB and WZ bulk GaAs as about $24 \mathrm{meV}$ per III-V pair at zero pressure [7]. It has been argued that this favoring of the ZB form might be offset in NWs of small diameter by the large relative contribution to the total energy of either the lateral facets 12 or the vertical edges separating the latter [13] (provided the specific energies of these features are less for WZ than for ZB). This naturally leads to critical radii under which WZ NWs should be more stable than ZB NWs. For instance, Akiyama et al. calculated a critical radius of 5.6 $\mathrm{nm}$ for GaAs NWs. These approaches have in common to treat the energetics of fully formed NWs and to predict critical radii far too small to explain the occurrence of WZ in NWs with radii up to at least $100 \mathrm{~nm}$.

On the other hand, from the very beginnings of VLS studies, it has been argued that the two-dimensional (2D) nucleation of new solid layers from the supersaturated liquid was of paramount importance [14] and most theories of NW growth take nucleation into account [15, 16, 17]. The fact that the faults in each phase and those separating ZB and WZ regions are perpendicular to the growth axis, in other words that each monolayer (ML) of III$\mathrm{V}$ pairs is uniform in structure and orientation, strongly suggests that, once a nucleus of critical size is formed, it rapidly spreads out laterally over the whole solid/liquid (SL) interface [5, 11], unless the wire is very wide. If so, the reason for the formation of the $\mathrm{WZ}$ phase should not be searched in the energetics of fully formed NWs [12, 13] but instead in the preferential formation of WZ nuclei at the SL interface. This is the aim of the present work.

Given the prominence of WZ in GaAs NWs, it is interesting to find two instances where the $\mathrm{ZB}$ structure systematically appears. The first one corresponds to the initial stage of NW growth. By growing GaAs NWs for short times, we ensured that the foot of each NW could be observed clearly before being buried by the $2 \mathrm{D}$ layer which grows concomitantly albeit more slowly between the NWs. The cross-sectional images obtained ex situ by transmission electron microscopy (TEM) prove that the whole NW is initially pure ZB. Growth then switches abruptly to WZ stacking (Fig. 11). Scanning electron microscopy shows that, at this early stage, the NWs are pyramids with triangular bases and tilted lateral facets. These become vertical at the $\mathrm{ZB} \rightarrow \mathrm{WZ}$ transition (Fig. 1).

The second observation is that when we terminate 


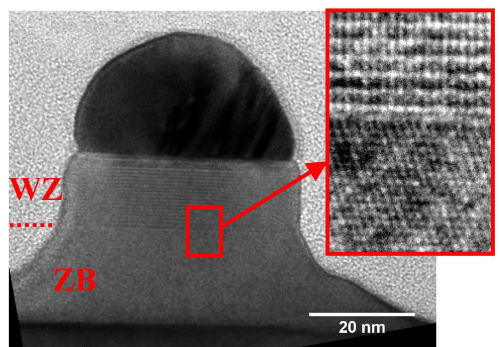

FIG. 1: (Color online) TEM image of a short GaAs nanowire with high resolution close-up of the $\mathrm{ZB} \rightarrow \mathrm{WZ}$ transition zone.

MBE growth by switching off the Ga flux while maintaining an As flux, a section of NW grows that systematically adopts the ZB structure. This effect, already reported for GaAs NWs grown by chemical beam epitaxy [3], is interpreted as a partial consumption of the Ga dissolved in the gold particle to form the terminal section of the NW.

These two situations where ZB forms have in common to be transient growth phases during which the supersaturation of Ga (and possibly As) in the liquid is less than during steady NW growth. Before growth, the deposited $\mathrm{Au}$ droplets dissolve the substrate locally to achieve equilibrium with it: the supersaturation is zero. When vapor fluxes are turned on, the supersaturation increases until a permanent regime settles. Symmetrically, during growth termination, the Ga concentration in the droplet, and hence supersaturation, decrease, since the atoms used to build the NW are not replaced. This strongly suggests that ZB systematically forms when the supersaturation is less than some critical value and, conversely, that WZ formation requires a high supersaturation.

This confirms the importance of nucleation. Indeed, according to nucleation theory, the work needed to form solid nuclei from a fluid phase is maximum for a critical nucleus size [18]. If this energy barrier is less for a certain crystal structure than for another, the first may nucleate preferentially even if the second one is more stable in bulk form. Since critical sizes and energy barriers scale inversely with supersaturation, our observations point to a preferential formation of $\mathrm{WZ}$ when the critical nuclei are small. This recalls the preference for WZ of NWs of small radius [12, 13] but we cannot simply assume it to hold for nuclei. Instead, we should compare the probabilities of forming various nuclei from the liquid phase. As a first approximation, we shall compare their formation energies, which largely determine these probabilities. In order not to obscure our demonstration, we keep as far as possible to continuous nucleation theory (CNT) [18], resorting to an atomistic picture only when necessary. We proceed in two stages. We first show that nucleation must occur at the edge of the SL interface (the triple solid/liquid/vapor line) rather than elsewhere in the SL interface. We then show that, along this line, the formation of WZ nuclei may be favored over that of ZB nuclei.
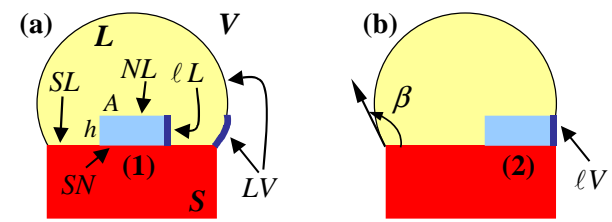

FIG. 2: (Color online) (a) Nucleus at the NW/liquid interface, with interfaces of interest. (b) Transferring the nucleus to the triple line eliminates and creates interfacial areas (thick lines).

Let us consider the interface between a NW (hereafter called substrate) and the liquid droplet. According to CNT, forming there a solid epitaxial 2D island of height $h$ (1 ML), perimeter length $P$ and upper area $A$ (Fig. 2(a)) from the liquid phase, involves a change of free enthalpy:

$$
\Delta G=-A h \Delta \mu+P h \gamma_{\ell L}+A\left(\gamma_{N L}-\gamma_{S L}+\gamma_{S N}\right)
$$

In Eq. (11), $\Delta \mu>0$ is the difference of chemical potential for III-V pairs between liquid and solid phases, per unit volume of nucleus; $\gamma_{\ell L}$ is the energy per unit area of the lateral interface between nucleus and liquid; $\gamma_{S L}, \gamma_{S N}$ and $\gamma_{N L}$ are, respectively, the energies per unit area of the substrate/liquid, substrate/nucleus (SN) and upper nucleus/liquid (NL) interfaces (Fig. 2(a)).

A given nucleus (set of atoms with fixed relative positions) of ML height cannot be said to be of $\mathrm{ZB}$ or $\mathrm{WZ}$ structure. It is only the orientational positioning of the nucleus with respect to the previous ML which determines if the stack of 2 MLs formed by adding the nucleus is of the type found in ZB or WZ crystals (Fig. 3). In the former case (hereafter 'ZB position') the $\mathrm{GaAs}_{4}$ tetrahedra have the same orientation if the Ga atom belongs either to nucleus or to previous ML whereas tetrahedra and nucleus are rotated by an odd multiple of $\pi / 3$ in the latter case ('WZ position') [7]. ZB and WZ sequences require the nucleation of each $\mathrm{ML}$ in, respectively, ZB and WZ position with respect to the previous $M L$.

Whatever the position of the nucleus, interfaces SL and NL have the same atomic configuration (Fig. 3) so that $\gamma_{S L}=\gamma_{N L}$. On the other hand, nucleation in WZ position costs some SN interfacial energy $\left(\gamma_{S N}^{W Z}=\gamma_{F}>0\right)$ whereas nucleation in ZB position does not $\left(\gamma_{S N}^{Z B}=0\right)$. Since forming a WZ sequence on a ZB substrate creates a SF, $\gamma_{F}$ is sometimes taken [5] as half the SF energy in

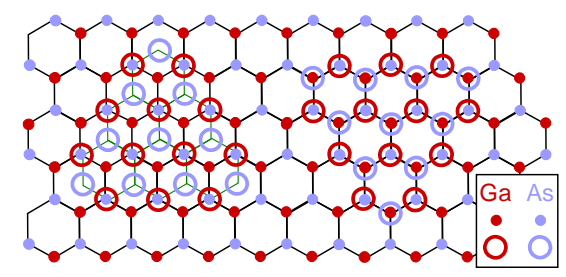

FIG. 3: (Color online) A given nucleus (open symbols) in ZB (left) and WZ (right) positions on top of a (111)B ML (disks). 
the bulk ZB phase [19]. Finally, from Eq. (1), the formation enthalpies of a given nucleus in $\mathrm{ZB}$ or $\mathrm{WZ}$ position are $\Delta G^{Z B, W Z}=-A h \Delta \mu+P h \gamma_{\ell L}+A \gamma_{S N}^{Z B, W Z}$. Since $\Delta G^{W Z}-\Delta G^{Z B}=A \gamma_{F}>0$, ZB nucleation is favored.

In the foregoing discussion, we did not specify if $\gamma_{\ell L}$ refers to ZB or WZ because the nucleus, which clearly has the same edges in both positions, was assumed to be laterally surrounded by the liquid. However, we now show that nucleation should take place at the triple line. Let us compare the formation of a given nucleus at two different locations (Fig. 21): its lateral surface is either entirely surrounded by the liquid (1) or partly surrounded by the vapor because of nucleation at the triple line (2). We now have to distinguish the specific energies $\gamma_{\ell L}$ and $\gamma_{\ell V}$ of the lateral nucleus/liquid and nucleus/vapor interfaces. The key point is that shifting the nucleus from location (1) to location (2) at constant liquid volume has a major effect: it eliminates part of the liquid/vapor interface and replaces it by nucleus/vapor interface (Fig. 2). Suppose that forming an area $s$ of nucleus/vapor interface eliminates an area $\tau s$ of liquid/vapor interface, of energy $\gamma_{L V}$. If $\alpha$ is the fraction of the island perimeter in contact with the vapor, the formation enthalpy of the nucleus now is:

$$
\begin{aligned}
\Delta G= & -A h \Delta \mu+P h\left[(1-\alpha) \gamma_{\ell L}+\alpha\left(\gamma_{\ell V}-\tau \gamma_{L V}\right)\right] \\
& +A \gamma_{S N}
\end{aligned}
$$

where, as above, $\gamma_{S N}=0$ or $\gamma_{F}$ for, respectively, ZB or WZ nuclei. For a given nucleus, the difference in formation enthalpies between locations (1) and (2) is $\Delta G_{2}-\Delta G_{1}=\alpha P h\left(\gamma_{\ell V}-\gamma_{\ell L}-\tau \gamma_{L V}\right)$. Factor $\tau$ cannot be calculated exactly. We estimate it by considering an artificially axisymmetric nucleus with a vertical lateral surface contacting the vapor along the whole triple line. This geometry preserves a spherical liquid/vapor interface. It readily yields $\tau=\sin \beta$, with $\beta$ the contact angle between droplet and substrate (Fig. 2). In all our samples, $90^{\circ} \leq \beta \leq 130^{\circ}$ (after growth) so that $0.85 \leq \tau \leq 1$.

Hence, a given nucleus tends to form at the triple line if $\gamma_{\ell V}-\gamma_{\ell L}-\tau \gamma_{L V}<0$, with $\tau \simeq 1$. Before growth, the contact angles between our liquid droplets and bulk GaAs are close to $\pi / 2$, which implies (from Young's equation) that the solid/liquid and solid/vapor interface energies are close for (111)B surfaces. Assuming that this also holds for the lateral nucleus faces yields $\gamma_{\ell L} \simeq \gamma_{\ell V}$. In turn, $\gamma_{L V}$ should lie between the surface energies of pure liquid $\mathrm{Au}$ and $\mathrm{Ga}$ (1.14 and $0.72 \mathrm{~J}^{\mathrm{m}} \mathrm{m}^{-2}$ [20]). Hence, the above inequality is safely satisfied and the critical nuclei should form at the edge of the droplet. In short, forming the nucleus there is advantageous because it eliminates a portion of the pre-existing droplet surface; this largely outweighs the replacement of part of the lateral nucleus/liquid interface by a possibly slightly costlier nucleus/vapor interface. Note that nucleation at the triple line in $\mathrm{GaP}$ NWs has previously been argued for on an entirely different basis, namely the low solubility of phos- phorus in gold [5]. Our argument is of general validity and would even apply to solid catalyst particles [3].

To demonstrate the advantage of WZ over ZB nucleation at the triple line, we consider the atomic structure of the edges of the top facet of the NW and of the nucleus. We simply select the low energy configurations discussed below by restricting ourselves to at most one non-vertical dangling bond per atom and to stoichiometric nuclei. At the first ZB $\rightarrow$ WZ transition (Fig. (1), given the pyramidal shape of the NW foot, the three edges of the top facet must be rows of $\mathrm{Ga}$ atoms. A given nucleus can again be placed there in $\mathrm{ZB}$ or $\mathrm{WZ}$ position, but this now entails major differences. The ZB nucleus must itself have a Ga edge at the triple line, so that the edges of the top NW facet and of the nucleus develop a tilted $\{111\} \mathrm{A}-$ type lateral facet (Fig. 4(a)). The WZ nucleus has an As edge at the triple line; the lateral facet is then vertical and of $\{10 \overline{1} 0\}$ WZ type (Fig. 4(b)). Since WZ sequences require the repeated nucleation of each ML in WZ position, the latter must therefore be favored at both Ga and As edges. This is also needed when WZ NWs with six $\{10 \overline{1} 0\}$-type vertical facets have started developing since their top facets have three edges of each type. However, at an As edge, ZB and WZ positions also differ: in ZB position, the nucleus itself has an As edge and generates a $\{111\}$ B-type tilted lateral facet (Fig. 4(c)), whereas in WZ position (not shown) it has a Ga edge and the lateral facet is again vertical, of $\{10 \overline{1} 0\} \mathrm{WZ}$ type.

Let us first compute the energy changes $\Delta_{j}$ upon transferring a given nucleus from location (1) (Fig. 2(a)) to three possible locations at the triple line (Fig. 4), one in WZ position with a lateral $\{10 \overline{1} 0\}$ facet $(j=W)$ and two in $\mathrm{ZB}$ position with lateral $\{111\} \mathrm{A}(j=A)$ or $\{111\} \mathrm{B}$ $(j=B)$ facets. When the lateral facets are tilted toward $(j=A)$ or away from $(j=B)$ the NW axis, the transfers also modify the areas of the solid/liquid and liquid/vapor interfaces, at fixed liquid volume. This can again be estimated by first considering toroidal nuclei preserving spherical liquid/vapor and axisymmetric SL interfaces, and then taking $\Delta_{j}$ as proportional to the fraction $\alpha P$ of

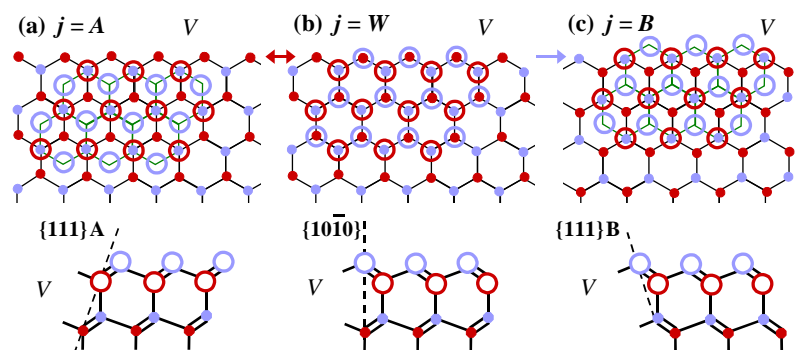

FIG. 4: (Color online) Top (top row) and side (bottom row) views of a given nucleus in $\mathrm{ZB}(\mathrm{a})$ and $\mathrm{WZ}$ (b) positions at a Ga edge and in ZB position at an As edge (c). Edges are indicated by arrows (top), the vapor side by $V$ and non-vertical dangling bonds by segments (bottom). Key as in Fig. 3 
the perimeter of the actual nucleus in contact with the vapor. We find $\Delta_{j}=\alpha P h\left(\widetilde{\gamma}_{j}-\gamma_{\ell L}-\gamma_{L V} \sin \beta\right)$ where $\widetilde{\gamma}_{j}=\gamma_{j} / \cos \theta_{j}+\left(\gamma_{L S}+\gamma_{L V} \cos \beta\right) \sin \theta_{j}$, with $\gamma_{j}$ the interface energy between vapor and lateral facet and $\theta_{j}$ the angle of the latter with the vertical $\left(\theta_{W}=0, \theta_{B}=-\theta_{A}=\right.$ $\left.19.5^{\circ}\right)$. Our $\widetilde{\gamma}_{j}$ have the same expression as the energies calculated by Ross et al. in a 2D model of facetted NWs [21], but they pertain to ML high nuclei.

Although it is not essential, we now specify that the nuclei are equilateral triangles of side $D$, one of them at the triple line $(\alpha=1 / 3)$. Their formation enthalpies are:

$$
\begin{aligned}
\Delta G_{j}= & -\frac{\sqrt{3}}{2} D^{2} h \Delta \mu+3 D h \gamma_{\ell L}-D h\left(\gamma_{\ell L}+\gamma_{L V} \sin \beta\right) \\
& +D h \widetilde{\gamma}_{j}+\frac{\sqrt{3}}{2} D^{2} h \gamma_{S N}
\end{aligned}
$$

Only the last two terms differ between nuclei and the last one is non-zero only in WZ position. Maximizing $\Delta G_{j}$ with respect to $D$ yields the critical size $D_{j}^{\star}$ and the energy barriers $\Delta G_{j}^{\star}=\Delta G_{j}\left(D_{j}^{\star}\right)$ for each nucleus:

$$
\Delta G_{W}^{\star}=\frac{\sqrt{3}}{6} \frac{\Gamma_{W}^{2}}{\Delta \mu-\frac{\gamma_{F}}{h}}, \Delta G_{k}^{\star}=\frac{\sqrt{3}}{6} \frac{\Gamma_{k}^{2}}{\Delta \mu} \text { for } k=A, B(4)
$$

where $\Gamma_{j}=2 \gamma_{\ell L}+\widetilde{\gamma}_{j}-\gamma_{L V} \sin \beta$ is an effective edge energy. WZ nuclei dominate if $\Delta G_{W}^{\star}<\Delta G_{k}^{\star}$ for $k=$ $A, B$. This requires two conditions. The first one, $\widetilde{\gamma}_{W}<$ $\widetilde{\gamma}_{k}$ for $k=A, B$, is material-related and involves only interface energies. A second, growth-related, condition is that the supersaturation be larger than a critical value, $\Delta \mu^{\star}=\max _{k=A, B}\left(\frac{\Gamma_{k}^{2}}{\Gamma_{k}^{2}-\Gamma_{W}^{2}} \frac{\gamma_{F}}{h}\right)$, to overcome the SF.

As a first approximation, we estimate the ZB $\widetilde{\gamma}_{j}$ energies from those of (111)A and B surfaces computed for As-rich (MBE) vapors, namely 0.82 and $0.69 \mathrm{~J}^{-\mathrm{m}^{-2}}$ 22]. In the extreme cases of drop surfaces of pure Ga (surface segregation of the low energy atom) and pure $\mathrm{Au}$, this gives respectively $\widetilde{\gamma}_{A}=0.76$ and $0.83 \mathrm{~J} \cdot \mathrm{m}^{-2}$ and $\widetilde{\gamma}_{B}=0.84$ and $0.77 \mathrm{~J}^{-\mathrm{m}^{-2}}$, well above the low $\gamma_{111 B}$ energy. Conversely, $\widetilde{\gamma}_{W}=\gamma_{10 \overline{1} 0}$ is unknown for As-rich vapors. According to the previous discussion, WZ forms because $\widetilde{\gamma}_{W}<\widetilde{\gamma}_{A}$ and $\widetilde{\gamma}_{B}$. This hypothesis is strengthened by calculating the critical supersaturations for a plausible range of such values, $0.7 \leq \widetilde{\gamma}_{W} \leq 0.75 \mathrm{~J} \mathrm{~m}^{-2}$. For e.g. a Ga drop surface and $\beta=120^{\circ}, \Delta \mu^{\star}$ ranges between 230 and $1570 \mathrm{meV}$, which is indeed of the order of our experimental supersaturations (several $100 \mathrm{meV}$ ).

In summary, we developed a nucleation-based model to explain the occurrence of the WZ phase in nanowires of ZB semiconductors, at least at certain stages of growth. A key and general result is that 2D nucleation takes place preferentially at the edge of the solid/liquid interface. When formed at this triple line, WZ and ZB nuclei present major differences and WZ nucleation is actually favored for certain ranges of the interface energies involved. In addition, the supersaturation of the liquid must be high enough, in agreement with our experimental results. Our aim was to identify important effects and parameters, not yet to give a complete description of the complex interplay of the two phases. We now intend to calculate the actual nucleation probabilities (including the effects of temperature and geometry), evaluate more precisely the energies of various nuclei (including non-stoichiometric ones) forming on NWs with different cross-sections, and take into account growth conditions in more details, in particular the supersaturation of each atomic species which appears here only indirectly via surface energies.

This work was partly supported by the SANDIE Network of Excellence of the European Commission (Contract No. NMP4-CT-2004-500101).

* Electronic address: frank.glas@lpn.cnrs.fr

[1] M. Koguchi, H. Kakibayashi, M. Yasawa, K. Hiruma, and T. Katsuyama, Jpn. J. Appl Phys. 31, 2061 (1992).

[2] X. Duan and C. M. Lieber, Adv. Mater. 12, 298 (2000).

[3] A. I. Persson, M. W. Larsson, S. Stenstrom, B. J. Ohlsson, L. Samuelson, and L. R. Wallenberg, Nature Mater. 3, 678 (2004).

[4] J. C. Harmand, G. Patriarche, N. Péré-Laperne, M.-N. Mérat-Combes, L. Travers, and F. Glas, Appl. Phys. Lett. 87, 203101 (2005).

[5] J. Johansson, L. S. Karlsson, C. P. T. Svensson, T. Mårtensson, B. A. Wacaser, K. Deppert, L. Samuelson, and W. Seifert, Nature Mater. 5, 574 (2006).

[6] R. S. Wagner and W. C. Ellis, Appl. Phys. Lett. 4, 89 (1964).

[7] C.-Y. Yeh, Z. W. Lu, S. Froyen, and A. Zunger, Phys. Rev. B 46, 10086 (1992).

[8] G. J. Ackland, Rep. Progr. Phys. 64, 483 (2001).

[9] M. I. McMahon and R. J. Nelmes, Phys. Rev. Lett. 95, 215505 (2005).

[10] P. Mohan, J. Motohisa, and T. Fukui, Nanotechnology 16, 2903 (2005).

[11] I. P. Soshnikov, G. E. Cirlin, A. A. Tonkikh, Y. B. Samsonenko, V. G. Dubovskii, V. M. Ustinov, O. M. Gorbenko, D. Litvinov, and D. Gerthsen, Phys. Sol. State 47, 2121 (2005).

[12] R. Leitsmann and F. Bechstedt (2006), condmat/0611521.

[13] T. Akiyama, K. Sano, K. Nakamura, and T. Ito, Jpn. J. Appl. Phys. 45, L275 (2006).

[14] B. Mutaftschiev, R. Kern, and C. Georges, Phys. Lett. 16, 32 (1965).

[15] E. I. Givargizov, J. Cryst. Growth 31, 20 (1975).

[16] V. G. Dubrovskii and N. V. Sibirev, Phys. Rev. E 70, 031604 (2004).

[17] D. Kashchiev, Cryst. Growth and Design 6, 1154 (2006).

[18] D. Kashchiev, Nucleation (Elsevier, Oxford, 2000).

[19] S. Takeuchi and K. Suzuki, phys. stat. sol. (a) 171, 99 (1999).

[20] A. Zangwill, Physics at surfaces (Cambridge University Press, Cambridge, 1988).

[21] F. M. Ross, J. Tersoff, and M. C. Reuter, Phys. Rev. 
Lett. 95, 146104 (2005).

B 54, 8844 (1996).

[22] N. Moll, A. Kley, E. Pehlke, and M. Scheffler, Phys. Rev. 